\title{
Diseño de un modelo de gestión ambiental a partir de la ISO 14001 para cuatro instituciones educativas del distrito de Buenaventura
}

\author{
Herick Grijalba Lerma ${ }^{\mathrm{a}}$, Bairon Martínez Vidal ${ }^{\mathrm{a}}$, Rafael Oyaga Martínez ${ }^{\mathrm{b}}$ \\ Faculta de Educación, Universidad Popular del Cesar, Aguachica, Cesar, Colombia. \\ hgrijalbalerma@gmail.com \\ Facultad de Ingeniería, Corporación Universitaria Reformada, Barranquilla, Colombia. \\ r.oyaga@unireformada.edu.co
}

Recibido: Agosto 24, 2021.

Recibido en su versión corregida: Octubre 21, 2021.

https://doi.org/10.54606/Sextante2021.v25.06

Aceptación: Octubre 29, 2021.

Cómo citar: Grijalba Lerma, H., Martínez Vidal, B. \& Oyaga Martínez, R. (2021). Diseño de un modelo de gestión ambiental a partir de la ISO 14001 para cuatro instituciones educativas del distrito de Buenaventura. Revista Sextante, 25, pp. 52 - 68, 2021.

\section{Resumen}

Se reporta el trabajo de investigación realizado en el distrito de Buenaventura en cuatro instituciones educativas, el cual tuvo como objetivo implementar la Norma ISO 14001 a través de la construcción de indicadores de gestión ambiental. Se concluyó que se determinó que el 100\% de las cuatro instituciones en las cuales se desarrolló la investigación, presentan serios problemas en la gestión de los residuos, igualmente lo centros educativos presentan deterioro en sus instalaciones físicas, y un sistema deficiente en acueducto, aseo y alcantarillado; y existe fragmentación en el liderazgo de la gestión ambiental. Además se encontró que la implementación de la ISO 14001 en las instituciones presenta la dificultad de la estructura organizativa de los centros escolares en Colombia, que muchas veces son diferentes a la organización empresarial, las escuelas se distancian en su administración y propuesta del concepto empresarial, a pesar de que en muchos sectores se habla de empresas educativas, a nivel público la estructura organizativa tiene unas particularidades como son: dependencia centralizada de las secretarias de educación, lo cual limita las decisiones, procesos y el manejo de los rubros destinados a fortalecer la gestión ambiental en las instituciones educativas (en las instituciones no existen rubros asignados a los programas de educación ambiental), porque tienen una responsabilidad en la formación integral de sus estudiantes, aspecto que redunda en que los procesos deben ir más allá de la mera gestión e involucrar acciones formativas. Y tienen un factor adicional muy importante, que son los actores (padres de familia) que juegan un papel fundamental en los procesos complementarios de formación y se depende de su participación (activa o apática) la capacidad de convocatoria institucional y el respaldo que le den al colegio.

Palabras clave: Educación ambiente; Desarrollo sostenible; Gestión ambiental; Recursos naturales.

\section{Drying of typical products from the Colombian Caribbean Coast under the analysis of the dryer performance index}

\begin{abstract}
The research work carried out in the district of Buenaventura in four educational institutions is reported, which aimed to implement the ISO 14001 Standard through the construction of environmental management indicators. It was concluded that it was determined that $100 \%$ of the institutions in which the research was developed have serious problems in waste management, likewise the four institutions have deterioration in their physical facilities, and a deficient system in aqueduct, toilet, and sewerage. there is fragmentation in the leadership of environmental management, it was found that the implementation of ISO 14001 in the four institutions presents the difficulty of the organizational structure of schools in Colombia, which are often different from the business organization, schools distance themselves in their administration and proposal of the business concept, although in many sectors there is talk of educational companies, at the public level the organizational structure has some particularities such as Centralized dependence of the secretaries of education, which limits the decisions and processes and the management of the items aimed at strengthening environmental management in educational institutions (In the four institutions there are no items
\end{abstract}


assigned to environmental education programs) because educational institutions have a responsibility in the integral training of their students for which, the processes must go beyond mere management and involve training actions and have a very important additional factor that are the parent actors who play a fundamental role in the complementary training processes and depends on their participation (active or apathetic) the capacity of institutional convocation and the support they give to the school Keywords: Environmental management, environmental education, sustainable development, natural resources.

Keywords: Environmental education; Environmental management; Natural resources; Sustainable development.

\section{Introducción}

En las instituciones educativas de Buenaventura se hace necesario la implementación del modelo de gestión ambiental bajo norma ISO 14001 porque va a permitir aunar esfuerzos con el PRAE, para mejorar las políticas ambiéntales institucionales, lo cual se traduce en el empoderamiento de los estudiantes y comunidad educativa en general frente a la ecoeficiencia, permitiendo la optimización y el uso adecuado de sus recursos. González (1996) plantea que es importante que la educación ambiental sea transversal y que relacione a todas las disciplinas con el propósito de generar una conciencia ambiental, no solamente en las instituciones, sino también en la sociedad en general.

Los sistemas educativos de la mayoría de los países incluyen entre sus objetivos rectores: el desarrollo de la educación ambiental como componente esencial de la formación integral de los estudiantes sobre la base de las relaciones interdisciplinarias en el currículo de los diferentes niveles educativos, en atención a los pronunciamientos de la Organización de las Naciones Unidas para la educación, la ciencia y la cultura, al plantear que el enfoque interdisciplinario es una necesidad para el abordaje de problemas ambientales (Henao Hueso \& Sanchez Arce, 2019).

\section{El problema de la investigación}

El objetivo de la educación ambiental es ser solución a las diferentes problemáticas ambientales, no es solo comprender los distintos elementos que componen el medio ambiente y las relaciones que se establecen entre ellos, sino también la adquisición de valores y comportamientos necesarios para afrontar los problemas ambientales actuales, acercándose a la idea de un desarrollo sostenible que garantice las necesidades de las generaciones actuales y futuras (Valdivieso, 2019).

En esta línea se deben impulsar la adquisición de la conciencia, los valores y los comportamientos que favorezcan la participación efectiva de la población en el proceso de toma de decisiones, debe señalarse, de tal manera que sea un medio de participación y que propicie la integración de todos los factores, que la educación ambiental se ha convertido hoy en día en uno de los mejores instrumentos para la correcta orientación de los valores y las conductas humanas para con el ambiente (Avila, 2016).

Ejemplo de ello son los PRAE que se establecieron desde el año 1994 a través de la Ley General de Educación, reglamentados el mismo año con el Decreto 1743 y reafirmados con la Política Nacional de Educación Ambiental (PNEA) del 2002, y con la Ley 1594 del 2012.

Como sabemos, los Proyectos Ambientales Escolares (PRAE) son en gran medida una respuesta y un mayor acercamiento a lo que son los Proyectos Educativos Institucionales (PEI) e impactar a los estudiantes con un aprendizaje significativo que contribuya al desarrollo de competencias ciudadanas y de pensamiento científico para el fortalecimiento de los procesos de gestión ambiental. (Jaramillo Carrillo et al., 2017).

Los Proyectos Ambientales Escolares a nivel nacional se han centrado en campañas de aseo, en siembra de árboles, en acciones de sensibilización, la celebración de fechas especiales según el calendario ambiental, huertas escolares, talleres de formación con enfoque conservacionista, entre otras acciones enfocadas desde los seis ejes definidos por la política nacional de educación ambiental (Jaramillo Carrillo et al., 2017). 
El diseño de un modelo de gestión ambiental a partir de la ISO 14001 en cuatro instituciones educativas públicas del distrito de Buenaventura, permitirá fortalecer los proyectos ambientales escolares (PRAES), puesto que como se referencia en los párrafos anteriores, los PRAES se han limitado frente al objeto o finalidad del mismo, logrando mínimos resultados, por lo que carecen de una política de gestión ambiental en las instituciones educativas, repercutiendo en un insuficiente manejo de los residuos sólidos, como también en el consumo irresponsable de agua y energía.

Teniendo en cuenta lo anterior, la meta de estos centros educativos debe ser lograr un adecuado aprovechamiento de sus recursos, con el convencimiento de obtener una sostenibilidad ambiental, mitigando los impactos generados por sus actividades diarias, y ser un ejemplo en el distrito de Buenaventura en lo concerniente a la implementación de políticas de gestión ambiental.

Por ello es pertinente y necesario que los entornos educativos cuenten con un modelo de gestión ambiental idónea que les permita ser coherentes con las políticas del orden nacional, regional y local, logrando así insertar los objetivos de desarrollo sostenible (ODS):

ODS 3. Garantizar una vida sana y promover el bienestar para todos en todas las edades.

ODS 4. Garantizar una educación de calidad inclusiva, equitativa, de calidad y promover oportunidades de aprendizaje durante toda la vida.

ODS 11. Lograr que las ciudades y los asentamientos humanos sean inclusivos, seguros, resilientes y sostenibles.

ODS 12. Garantizar modalidades de consumo y producción sostenibles.

Es de vital importancia la construcción de indicadores ambientales como complemento al modelo de gestión, lo cual va a permitir realizar seguimiento y evaluación a los PRAES en relación con la puesta en marcha del modelo de gestión propiamente dicho, ver si esta estrategia contribuye al fortalecimiento de los proyectos ambientales escolares institucionales en lo concerniente a la transversalidad como pilar fundamental en el sostenimiento del sistema (Oyaga, 2011).

Sobre la estrategia ambiental, podemos mencionar que se establece como un plan cuyo fin es mitigar los impactos ambientales negativos, que pudieran ser generados por las operaciones de una organización. Estos impactos ambientales incluyen aquellos relacionados con el uso y agotamiento de los recursos naturales, emisiones atmosféricas, vertimientos líquidos, ruidos, disposición inadecuada de residuos, entre otros (Torres Salas, 2015).

El interrogante sobre la gestión ambiental se ha propuesto desde la perspectiva de si es un sistema de prevención o de reparación, quizá sea demasiado evidente que esto depende del entorno donde se desarrolla. Esto se debe al sistema económico que nos impera, ya que es mejor contaminar y luego reparar, por ello es necesario entender cuál es la discusión, si es que faltan leyes que reglamenten el uso de los recursos naturales o cambiar de sistema o modelo económico que si tenga en cuenta el medio ambiente.

\section{Ubicación geográfica}

Buenaventura es la ciudad más importante en el pacífico colombiano y el municipio de mayor extensión de él departamento del Valle del Cauca. Ubicada a $115 \mathrm{~km}$ por carretera de Cali, separada por la Cordillera Occidental de los Andes. Habitan 342,610 personas, la mayoría en la Zona urbana. El municipio está integrado por 20 corregimientos. La ciudad está dividida en 12 comunas (165 barrios), de las cuales cuatro pertenecen a la zona insular y ocho a la zona continental. Es el puerto marítimo más importante sobre el Océano Pacífico y el más importante de Colombia por su volumen de carga. $80 \%$ del café es enviado al exterior a través de su puerto y $40 \%$ de todo el comercio internacional marítimo de Colombia.

Las instituciones educativas se encuentran ubicadas en la zona urbana, las cuatro instituciones están ubicadas en la comuna 8 (ver Figura 1), continente del distrito especial y biodiverso de Buenaventura, con estratificación socioeconómica 3 al igual que una población predominantemente afrodescendiente, cuentan con canchas de fútbol, 
basquetbol, zonas verdes, cafetería, sala de sistemas, entre otros lugares.

La Institución Educativa Las Américas se encuentra ubicada en la dirección Cra. $65 \mathrm{~b} \# 6^{\mathrm{a}}-26$, barrio Las Américas, zona continente de la ciudad de Buenaventura, con cerca de dos mil estudiantes.

La Institución Educativa Simón Bolívar se encuentra ubicada en la dirección cra 60 y 61, barrio El Dorado, zona continente de la ciudad de Buenaventura, con cerca de 2.300 estudiantes.

La Institución Educativa José María Cabal se encuentra ubicada en la dirección 62c-48, calle 11 , barrio Independencia, zona continente de la ciudad de Buenaventura. Tiene aproximadamente 2.500 estudiantes.

La Institución Educativa Teófilo Roberto Potes se encuentra ubicada en la dirección Cra. 47 \# 5-17, barrio Bellavista, zona continente de la ciudad de Buenaventura. Esta tiene aproximadamente tres mil estudiantes.

Estas cuatro instituciones se encuentran ubicadas en barrios de estrato 2, con una población predominante de raza negra. La tasa de desempleo en Buenaventura es del 33\%, la tasa de desempleo juvenil es del $26 \%$. El $27 \%$ de los bonaerenses no cuenta con alcantarillado y el $39 \%$ sin alcantarillado.

\section{Marco conceptual}

En este apartado se ha realizado una amplia recolección de información de diferentes referentes teóricos que establecen la importancia de la implementación de sistemas de gestión ambiental, no solo en empresas, sino también en instituciones educativas, por ello es conveniente la aplicabilidad de esta proceso investigativo: Diseño de un modelo de Gestión Ambiental a partir de la ISO 14001 para cuatro Instituciones Educativas del distrito de Buenaventura, esto con el fin de promover un modelo que contemple las diferentes problemáticas ambientales en las instituciones educativas del Distrito de Buenaventura y dar solución a las mismas, por tal motivo se trae a colación a Nannett \& Leyva (2015) con un análisis del entorno para la

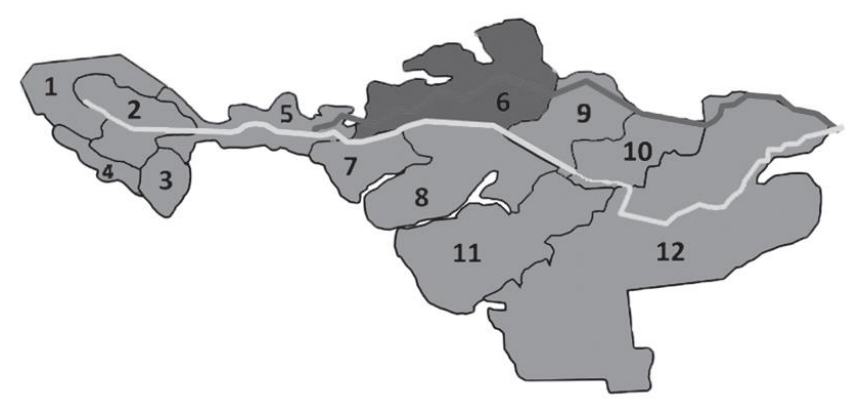

Figura 1. Barrios que conforman las comunas en Buenaventura.

Fuente: Los autores.

gestión ambiental en Colombia durante 20 años (1994-2014):

Hasta hace muy poco tiempo los bienes y servicios ecosistémicos fueron muy superiores a las necesidades de las sociedades humanas; para propósitos prácticos el mundo era ilimitado. Siempre existió la posibilidad de encontrar nuevos espacios y recursos. El dominio de la naturaleza expresado como la transformación de los ecosistemas a costa de la destrucción de las coberturas vegetales naturales, la eliminación de los animales silvestres, considerados enemigos potenciales, y la explotación de los recursos. La evolución del Ministerio de Ambiente de Colombia en sus primeros veinte años: 19942014 considera sus efectos sobre el medio ambiente, y se entendían como símbolos de progreso, al igual que el control $y$ encauzamiento de los ríos y la desecación de los humedales. (p.30).

Por tal motivo, desde esta perspectiva las empresas y las sociedades determinaban que los recursos naturales eran ilimitados según lo planteado y por tal motivo se realizaba un consumo excesivo de estos.

Hoy en día vivimos las consecuencias, no solo por la contaminación y la destrucción del ambiente, sino la falta de los recursos naturales, algunos minerales que se pensaban que eran finitos dejan de serlo y por fin llegamos a entender que hay recursos no renovables. Por ello es necesario generar un nuevo tipo de consumo más sostenible, partiendo del supuesto que es de vital importancia proveerle recursos a las generaciones futuras como lo mencionan los objetivos del desarrollo sostenible (ODS). De esta manera, la educación ambiental no 
puede ser limitada y por tanto debe ser transversal, con el fin de generar un impacto en los diferentes sectores de la sociedad.

Los sistemas de gestión ambiental no deben ser solo para las empresas, sino que deben ser herramientas y/o estrategias para la formación continua en todas las esferas sociales como lo menciona Massolo (2015), haciendo énfasis en la actualización constante que deben tener los sistemas de gestión ambiental. "Debido al carácter antropocéntrico, para la resolución de los problemas, es necesario tener en cuenta diversos factores relacionados a las ciencias sociales además de los relacionados a las ciencias naturales" (Massolo, 2015).

Los sistemas de gestión ambiental buscan introducirse no solo en las empresas, sino también en los centros educativos, ya sea por medio de instrumentos y herramientas de planeación, con el fin de generar una mejor calidad en la educación ambiental y así propiciar una protección y una defensa a los ecosistemas. Que no sea demasiado tarde para implementar un sistema de gestión ambiental capaz de dar solución o por lo menos mitigar los daños ambientales; en México, por ejemplo, su intervención frente a los sistemas de gestión ambiental sí son eficaces y efectivos según lo señalado por la Secretaria de Medio Ambiente y Recursos Naturales de ese país, ya que al ser uno de los países más contaminados del continente americano, según Hernández (2016), México está al borde de un colapso ambiental y en el límite de una crisis por el agua, por lo que ha adoptado mecanismos para evitar que se sigan presentando casos de contaminación.

Por otra parte, y teniendo en cuenta los sistemas de gestión ambiental Bibiloni (2008) expresa cómo los niveles administrativos, ya sean a nivel nacional, regional, o local, deben realizar una hoja de ruta para que el accionar ambiental sea eficiente y de respuesta a los problemas ambientales. Recalca también que se deben dejar las reflexiones de papel para dar paso a las acciones concretas que estimulen la conservación del ambiente, generando la necesidad de diseñar instrumentos que puedan ser implementados en las instituciones con el propósito de contribuir a un cambio en cómo abordar los problemas ambientales.
Entre tanto los autores (Martinez \& Figueroa, 2013), mencionan que en últimas los sistemas de gestión ambiental obedecen a términos más del entorno económico que de lo socio ambiental, parte de la definición histórica de la gestión ambiental:

La gestión ambiental se ha enfocado principalmente en la administración de los recursos naturales contenidos en unidades espaciales territoriales $\mathrm{o}$ ecológicas, asumiéndolos como un capital natural aprovechable, perteneciente a una organización determinada, pública o privada, bajo un enfoque predominante de sostenibilidad débil. Si bien se procura la integración de los sistemas natural, social y económico bajo una lógica lineal, esta relación intenta mantener o incrementar el capital total en una temporalidad limitada, situación que favorece el remplazo de los capitales interactuantes como el natural (Martínez \& Figueroa 2013, p. 15).

La educación ambiental es una compleja dimensión de la educación global, caracterizada por una gran diversidad de teorías y de prácticas, que abordan desde diferentes puntos de vista la concepción de educación, medio ambiente, desarrollo social y de educación ambiental (Bedoya Mocoso, 2015).

Por ello se hace necesario la implementación del sistema de gestión ambiental basado en la NTC ISO 14001, como herramienta idónea que coadyuva a los PRAES, permitiendo que las instituciones, estudiantes y comunidad educativa en general propendan por adquirir una cultura ambiental y de esa manera lograr comunidades sostenibles.

El sistema de gestión ambiental busca que las instituciones en todos sus procesos sean amigables con el ambiente, de allí que la implementación de un sistema de gestión ambiental puede ser un proceso transversal en las instituciones educativas que genere grandes oportunidades e impacte de manera positiva en la formación de los estudiantes, sus valores y relación con el entorno. El éxito está cuando se logra alinear el SGA con la estrategia pedagógica de la institución (Perez Ramirez, 2017).

La gestión ambiental se desarrolla con éxito en las organizaciones con bases teóricas y propuestas 
metodológicas concretas en el nivel empresarial (Isaac Rodríguez, 2012).

Hernández (2020), afirma que es perentorio que las instituciones educativas implementen el sistema de gestión ambiental, ya que este además permite minimizar los impactos negativos y logra imbuir a los estudiantes a que sus acciones y comportamientos sean siempre bajo el marco del desarrollo sostenible.

Teniendo en cuenta que la gestión ambiental, además de contener la protección del suelo, el aire y el manejo adecuado de los residuos sólidos, también trae consigo el buen manejo y uso racional del recurso hídrico. Es por ello por lo que para optimizar el uso del agua en una entidad educativa es necesario aplicar una estrategia basada en tres aspectos importantes: mejorar los niveles de conciencia ambiental de los usuarios, implementar mecanismos de control y establecer lineamientos claros relacionados y orientados al cuidado de dicho recurso (Torres Salas, 2015).

Oyaga (2011) plantea que no solo las empresas, sino también las instituciones educativas deben implementar procesos de gestión de carácter ambiental como lo son: los sistemas de gestión ambiental. Este autor presenta como ejemplo el desarrollo de las eco-auditorias escolares, entre otros mecanismos, con el propósito de generar una construcción de conocimiento ambiental:

Esto motivó la necesidad de construir e implementar una serie de indicadores de evaluación que permitieran evaluar los alcances que la estrategia didáctica de Eco auditoria escolar había generado dentro de los estados de los proyectos ambientales escolares, con el fin de determinar fortalezas y debilidades, y poder realizar un plan de mejora con el fin de construir una cultura ambiental al interior de las instituciones evaluadas (Oyaga, 2011).

Un indicador es una expresión cualitativa o cuantitativa observable, que permite describir características, comportamientos o fenómenos de la realidad a través de la evolución de una variable o el establecimiento de una relación entre variables (Diaz \& Meneses Silva, 2015).
Los indicadores ambientales han adquirido relevancia en los últimos años dada su capacidad de generar una imagen sintética de las condiciones ambientales del territorio. Su auge se ha desarrollado de forma paralela a los avances, acuerdos y retos ambientales a nivel global, lo que ha orientado la determinación de niveles permisibles, regulaciones y leyes por parte de las entidades encargadas del monitoreo y control ambiental (Muñoz Montilla, 2018). Por ello la pertinencia de incorporar los indicadores ambientales en esta investigación, permitiendo robustecer el modelo de gestión ambiental para que las instituciones puedan ser ecoeficientes minimizando sus impactos ambientales.

\section{Enfoque de la investigación}

El positivismo ha cumplido una excelente función formativa que no se puede desconocer. $\mathrm{Su}$ efecto pedagógico y didáctico ha educado generaciones de ciudadanos al transmitir el conocimiento en los espacios escolares (Rivera, 2020). De allí que esta investigación propende por fomentar en las instituciones educativas una cultura ambiental para la prevención y cuidado del ambiente como acción formativa en los estudiantes, de manera que la implementación del sistema de gestión ambiental en las 4 instituciones educativas cumple su enfoque positivista.

Es necesario abordarlo desde la formación de profesores, quienes a partir de su conocimiento ya sea desde un paradigma positivista o cultural, construyen los proyectos ambientales escolares (PRAE) en instituciones educativas a nivel básico y secundario (Cáceres, 2016). En ese sentido es de vital importancia abordar de manera transversal la educación ambiental en las 4 instituciones educativas del distrito de Buenaventura, ya que este sistema de gestión y los proyectos ambientales escolares permitirán que dichas instituciones puedan robustecer su política de educación ambiental, para lo cual contarán con un cuerpo docente idóneo y capacitado, procurando siempre la mejora continua del sistema.

El positivismo se ha orientado hacia el descubrimiento de las causas que generan los fenómenos del mundo social, considerados como objeto de estudio (Barona, 2020). En ese sentido es loable pensar que dicho enfoque es pertinente en 
esta investigación, porque las problemáticas sociales que se están viviendo en los planteles educativos son la falta o carencia de educación ambiental en los estudiantes, lo cual es nuestro objeto de estudio. Por esta razón se ve la necesidad de implementar el sistema de gestión como instrumento que va a permitir minimizar las causas que generan este flagelo o problemática social, que por ende nos atañe a todos.

En lo metodológico el positivismo se caracteriza por emplear la experimentación controlada, en la cual se establece un diseño riguroso y estructurado, en el que se emplea una metodología pensada para evitar los sesgos en la información y la subjetividad por parte del investigador.

De tal manera se afirma que la metodología en el paradigma cuantitativo o positivista, según (Prieto, 2012) es ordenada, intencional, controlada, deductiva, rigurosa, de tal manera que esta investigación, que es de carácter positivista, tiene todas estas directrices mencionadas, que le dan peso y soporte frente a los resultados que serán de carácter conativo.

En este sentido, el análisis estadístico que se realiza a los datos cuantitativos constituye una parte fundamental de la metodología positivista (León Pirela \& Pérez, 2019). De allí la importancia de un análisis exhaustivo y riguroso de los datos o la información que se va a procesar en la investigación. En ese orden de ideas, la investigación tendrá un análisis riguroso de la información recabada de las 4 instituciones educativas del distrito de Buenaventura.

El positivismo se inició como un modelo de investigación en las ciencias físicas o naturales y posteriormente se lo adoptó en el campo de las ciencias sociales (Ricoy, 2006). Esto es importante porque esta investigación tiene un carácter social y ambiental, iniciando por la gestión ambiental y finalizando con la formulación de recomendaciones sociales que den respuesta a las diferentes problemáticas de las instituciones educativas de la ciudad de Buenaventura.

Según Rodilla Gonzales \& Ramon Pascua (2006) "Permiten que el jurista positivista, en definitiva, una amplitud de criterio mayor y más moderno". Lo que permite afirmar que este tipo de investigación intenta dar posiciones claras y precisas frente al campo de estudio y dando como resultado un mayor criterio y nuevos aportes.

Esta investigación parte de una aproximación conceptual a los diferentes postulados sobre las investigaciones positivistas por ello se trae a colación el siguiente autor que menciona que: "El ideario positivista no concede en realidad espacio para un conflicto de las facultades entre filosofía y ciencia, el nombre de filosofía subsiste ciertamente pero entonces o en sinónimo de ciencia positiva o de síntesis de todas las ciencias positivas" (Gracia J, 2005).

Desde lo anterior se debe tener en cuenta que los métodos utilizados en la investigación positivista se tomarán principalmente de las ciencias naturales y consisten en la observación, experimentación y técnicas de encuestas (Schiffman L \& Kanuk L, 2005).

\subsection{Población y muestra}

Es importante mencionar que las cuatro instituciones educativas que se abordaran tienen claro que "La muestra debe ser representativa de la población que se desea estudiar y reflejar las características de los elementos que la componen" (Esteban, I\& Fernández, E, 2017).

De lo anterior se puede decir también que es de resaltar que en esta investigación no se parte de supuesto, sino de teorías y aproximaciones conceptuales, como lo mencionado a continuación, muestreo local utilizado para estudiar actitudes y opiniones en las que el criterio de selección de los sujetos obedece al lugar de residencia, este método de muestreo es considerado probabilístico pues la selección es aleatoria (Del río, S, 2013).

De tal manera que se tenga claro lo siguiente, en general no me interesan los datos de todos los individuos, de todo universo, sino que se estudian poblaciones, aun así, obtener y analizar los datos de toda la población suele ser imposible por lo que en la práctica suele seleccionar una muestra de individuos de la población (Tomás Sábado, J, 2010).

Esto quiere decir que no es importante el total de la población, sino una muestra que sea representativa y esta pueda ser generalizada. 
Por lo anterior, también se puede afirmar que la muestra tiene que ser seleccionada aleatoriamente, todos los elementos de la población antes de la selección de la muestra deben tener una probabilidad mayor que cero de ser incluidos en la muestra (Cáceres. R, 2007).

Existe cierto grado de muestreo probabilístico, lo que aumenta la representatividad de la muestra (Barrera, 2017). Por lo que es necesario y pertinente que todos los estudiantes tengan la misma probabilidad para la aplicación de la encuesta o participación de esta.

Los métodos probabilísticos para el pronóstico de la población están ganando reconocimiento rápidamente, ya que su principal ventaja radica en la llamada consistencia probabilística a través de todas las variables (Sacco \& Andreozzi, 2017). En consecuencia, se consideran de gran importancia las variables en la gestión ambiental, tales como reducción de residuos sólidos, uso adecuado de zonas verdes, reducción de niveles de ruido, uso racional de agua y la energía, como también la mejora de las instalaciones.

Esta investigación parte de la hipótesis de: si es posible establecer un modelo ambiental a partir de la ISO 14001, para cuatro instituciones educativas del distrito de Buenaventura, por lo anterior es importante mencionar que "La idea o el proyecto de investigación parte de objetos de la realidad factual y su pretensión en la solución de problemas prácticos.” (Rocha C. M., 2015).

\subsection{Variables}

Dependientes:

- Implementación de gestión ambiental.

Independientes:

- Satisfacción con el colegio.

Paradigma:

El paradigma cuantitativo ubicado dentro del positivismo y del análisis estadístico. Desde esta posición los resultados se expresan a través de indicadores y modelos estadísticos matemáticos, por lo tanto, dentro de dicho modelo se sigue un patrón predecible y estructurado. Se tiene presente que las decisiones críticas se efectúan antes de la recolección de datos. Se pretende generalizar los resultados encontrados en un grupo o segmento (Morales, 2018). Por lo que es de suma importancia establecer unos indicadores ambientales y de evaluación en este estudio.

Ricoy (2006) indica que el "paradigma positivista se califica de cuantitativo, empíricoanalítico, racionalista, sistemático gerencial $\mathrm{y}$ científico tecnológico". Por tanto, el paradigma positivista sustentará a la investigación que tenga como objetivo comprobar una hipótesis por medios estadísticos o determinar los parámetros de una determinada variable mediante la expresión numérica (Ramos, 2015). Por lo que, en esta investigación, además de comprobar la hipótesis, se establecerán las dimensiones (ver Tabla 1), lo cual va a permitir evaluar las variables establecidas en el estudio.

El paradigma de investigación positivista, también denominado cuantitativo o racionalista, predomina en muchas comunidades científicas. Este paradigma asume una concepción de la realidad única, objetiva y tangible, que puede ser fragmentada en partes, para su estudio.

Tradicionalmente la investigación en el ámbito educativo se ha visto influenciada por sus postulados y principios (Gil et al., 2017). Y esta investigación no es la excepción, partiendo de la aproximación conceptual de autores positivistas, de resultados cuantitativos. Esta investigación parte de ello, de datos y hechos reales llevados a estudio.

La realidad única y objetiva de esta investigación es la de permitir o establecer en los centros educativos del distrito de Buenaventura políticas de gestión ambiental de cara a los objetivos de desarrollo sostenibles (ODS).

De tal manera que este tipo de investigación tenga en cuenta que "En este método cuantitativo el saber científico se caracteriza por ser racional, objetivo, se basa en lo observable, en lo manipulable y verificable (Cuenya \& Ruetti,2010).

Los hallazgos basados en este paradigma son reales y generalizables a toda la población (Guba \& Lincoln, 2002). Por ello es importante escoger bien 
Tabla 1. Descripción de variables.

\begin{tabular}{ll}
\hline \multicolumn{1}{c}{ Dimensiones } & \multicolumn{1}{c}{ Variables } \\
\hline $\begin{array}{l}\text { Gestión ambiental } \\
\text { y de la institución } \\
\text { educativa }\end{array}$ & $\begin{array}{l}\text { Reducción de residuos sólidos. } \\
\text { Uso adecuado de zonas verdes. } \\
\text { Reducción niveles de ruido. } \\
\text { Uso racional del agua y la energía. } \\
\text { Mejora de las instalaciones. }\end{array}$ \\
\hline $\begin{array}{l}\text { Participación activa } \\
\text { de la comunidad } \\
\text { educativa }\end{array}$ & $\begin{array}{l}\text { Satisfacción con el colegio. } \\
\text { Inserción de la comunidad. }\end{array}$ \\
& $\begin{array}{l}\text { Generación de proyectos ambientales. } \\
\text { Valoración de lo común. }\end{array}$ \\
\hline $\begin{array}{l}\text { Estructura y } \\
\text { transversalización } \\
\text { curricular }\end{array}$ & $\begin{array}{l}\text { Fortalecimiento del PRAE. } \\
\text { Fortalecimiento de las estrategias } \\
\end{array}$ \\
Pedagógicas Ambientales. \\
Trabajo en equipo.
\end{tabular}

Fuente: Los autores.

Tabla 2. Descripción de categorías.

\begin{tabular}{|c|c|c|}
\hline Categorías & Descripción & Precategorías \\
\hline $\begin{array}{l}\text { Instrumentos para } \\
\text { la gestión } \\
\text { ambiental. }\end{array}$ & $\begin{array}{l}\text { Son los mecanismos de } \\
\text { implementación del } \\
\text { sistema ISO en las } \\
\text { instituciones } \\
\text { educativas. }\end{array}$ & $\begin{array}{l}\text { PRAE. } \\
\text { Calidad educativa. } \\
\text { Seguimiento. }\end{array}$ \\
\hline $\begin{array}{l}\text { Problemáticas } \\
\text { ambientales. }\end{array}$ & $\begin{array}{l}\text { Son los impactos } \\
\text { ambientales a los cuales } \\
\text { están sometidas las } \\
\text { instituciones } \\
\text { educativas. }\end{array}$ & $\begin{array}{l}\text { Residuos. } \\
\text { Ruido. } \\
\text { Olores. } \\
\text { Gestión de recursos. }\end{array}$ \\
\hline $\begin{array}{l}\text { Instituciones } \\
\text { educativas del } \\
\text { distrito de } \\
\text { Buenaventura. }\end{array}$ & $\begin{array}{l}\text { Características de las } \\
\text { comunidades } \\
\text { educativas donde se } \\
\text { implementan el } \\
\text { proyecto. }\end{array}$ & $\begin{array}{l}\text { Tamaño de la } \\
\text { población escolar. } \\
\text { Políticas } \\
\text { institucionales sobre } \\
\text { la gestión ambiental. }\end{array}$ \\
\hline
\end{tabular}

Fuente: Los autores.

la muestra, esto determinará que el resultado de la investigación sea posible generalizarlo.

Teniendo claro lo anterior y partiendo de esta investigación, es importante mencionar que: "El razonamiento cuantitativo mantiene el propósito de aplicar algunos tópicos matemáticos a situaciones de la vida cotidiana” (Rojas Álvarez C, 2018).

De esta manera es conveniente precisar que, "La elaboración del sistema productivo, pero también en otros contextos con situación de cierta complejidad que plantean problemas susceptibles de ser resueltos elaborando un modelo cuantitativo" (Sallan Leyes J, 2009). Por ello la investigación parte de un paradigma cuantitativo, ya que por medio de datos numéricos identificaremos diferentes problemáticas presentes en las instituciones educativas de la Ciudad de Buenaventura.
Esta investigación, siendo pionera en la región, esta soportada por diferentes autores, por ello es preciso decir que, "La educación es una actividad inherente al desarrollo del ser humano que le permite desplegar sus potencialidades y cultivar sus capacidades" (Tolley E, 2006).

\section{Técnicas e instrumentos de recolección de datos}

Para esta investigación, que es de carácter cuantitativo y no experimental, se utilizaron diferentes instrumentos de recolección de datos, por ello traemos a colación que: la utilización del método científico en la investigación educativa y social supone un intento sistemático dirigido a cuestionar y resolver problemas a través de la recogida y análisis de los datos primarios con el propósito de describir, explicar, generalizar y predecir (Catalina. M, \& Arturo. G, 2014).

Teniendo claro lo anterior y la información consolidad en las Tablas $\underline{2}$ y $\underline{3}$, esta investigación utilizará la observación, encuestas, datos, entre otros instrumentos de recolección, donde prime la veracidad y la claridad de los datos. Dado que el valor de una investigación depende de que la data obtenida refleje lo más fidedignamente lo que ocurre en el evento o fenómeno sujeto/objeto de estudio. Cobra mayor relevancia conocer los diversos tipos de instrumentos y en qué contextos pueden ser utilizados (Corral, 2014).

Término con lo siguiente, el instrumento de medición o de recolección de datos tiene un papel central, sin él no hay observaciones clasificadas (Sampieri. R, 2018).

Los instrumentos permiten transformar datos del mundo subjetivo y objetivarlos a través del contraste entre teoría y observación (Caparó García \& Duffaut, 2016). Por lo que la técnica para esta investigación será la encuesta y como instrumento se utilizará el cuestionario, siendo este un instrumento diseñado para cuantificar de manera sistemática la magnitud de la variable. Este instrumento permitirá una adecuada validación.

Otro instrumento para la recolección de datos que se aplicará en esta investigación es la observación, una técnica donde el observador puede 
Tabla 3. Descripción de variables.

\begin{tabular}{|c|c|c|c|}
\hline Variables & Dimensiones & Indicador & Subindicador \\
\hline $\begin{array}{l}\text { Gestión } \\
\text { ambiental } \\
\text { sostenible del } \\
\text { entorno } \\
\text { escolar. }\end{array}$ & $\begin{array}{l}\text { Tipo de vector: } \\
\text { Programa de } \\
\text { educación } \\
\text { ambiental. } \\
\text { Estrategias. } \\
\text { Indicadores de la } \\
\text { gestión. }\end{array}$ & $\begin{array}{l}\text { Revisión } \\
\text { periódica. } \\
\text { Evaluación } \\
\text { semestral. } \\
\text { Acciones de } \\
\text { mejora. }\end{array}$ & $\begin{array}{l}\text { Acciones de } \\
\text { las autoridades } \\
\text { institucionales } \\
\text { responsables. }\end{array}$ \\
\hline $\begin{array}{l}\text { Participación } \\
\text { de la } \\
\text { comunidad } \\
\text { educativa en } \\
\text { la gestión } \\
\text { ambiental. }\end{array}$ & $\begin{array}{l}\text { Participación en } \\
\text { reuniones sobre la } \\
\text { gestión ambiental. } \\
\text { Comité ambiental. }\end{array}$ & $\begin{array}{l}\text { Número de } \\
\text { reuniones. } \\
\text { Tiempo de } \\
\text { convocatoria. }\end{array}$ & $\begin{array}{l}\text { Evidencias en } \\
\text { documentos. }\end{array}$ \\
\hline $\begin{array}{l}\text { Estructura } \\
\text { curricular. }\end{array}$ & $\begin{array}{l}\text { Transversalización } \\
\text { de la educación } \\
\text { ambiental. }\end{array}$ & $\begin{array}{l}\text { Asignaturas } \\
\text { presentes. }\end{array}$ & $\begin{array}{l}\text { Programas de } \\
\text { educación } \\
\text { ambiental. }\end{array}$ \\
\hline PRAES. & Existencia de este. & $\begin{array}{l}\text { Numero de } \\
\text { áreas } \\
\text { responsable. }\end{array}$ & $\begin{array}{l}\text { Situación del } \\
\text { PRAES. }\end{array}$ \\
\hline
\end{tabular}

Fuente: Los autores.

describir los sucesos exactos que están pasando en el momento de aplicar la técnica (Gómez, 2016).

Se propone un modelo para la recolección de datos, con el fin de reconocer los indicadores que permiten evaluar la calidad de los servicios (Vargas, 2015). De allí que esta investigación contempla los indicadores ambientales y los de evaluación, lo que permite medir los impactos.

\subsection{Observación}

Este apartado se basa en observar de forma sistemática algún hecho o acontecimiento con relación a los objetivos de la investigación. Estos pueden integrarse como participante $o$ no participante, donde el primero refiere al fin donde el investigador hace parte de la población de muestreo; y el segundo es donde el investigador no se vincula en el medio en el que se realiza el estudio. En adición puede clasificarse en estructurada y no estructurada. En la primera se utiliza un patrón que especifica qué elementos serán observados y la segunda refiere a no contar con un patrón previa a su realización (Arias, 2012).

Con respecto a los instrumentos utilizados para la observación libre se encuentran: las anotaciones y el diario de campo, además de cámaras fotográficas o de video.

Para la observación más estructurada se usan instrumentos que se diseñan previamente, como las frecuencias, escalas y listas de cotejo (Arias, 2012).
Por su parte Sierra y Bravo (1984), la define como: "La inspección y estudio realizado por el investigador, mediante el empleo de sus propios sentidos, con o sin ayuda de aparatos técnicos, de las cosas o hechos de interés social, tal como son o tienen lugar espontáneamente”. (p.34)

Maestre (1989) expone que consiste en la introducción del investigador en la comunidad o grupo que desea investigar y, "a base de integrarse como un miembro más de esa sociedad poder obtener los datos deseados", lo cual implica la necesidad de participar en la vida comunitaria y observará lo que sucede a su alrededor (p.12).

La observación es un proceso sistemático que permite a quien lo realice, detectar peculiaridades dentro de un entorno o proceso determinado. La observación es también un método empleado en diferentes campos del trabajo investigativo. De acuerdo con Goetz y Le compte (1988), beneficia de igual manera la ocupación de cualquier persona que lleva a cabo sus funciones de supervisión, ya que le atribuye, desde una postura independiente $\mathrm{y}$ detallada, rehacer las características del sujeto observado. Esta definición concuerda con la expresada por Valdez (2000) quien indica que:

La observación es un método empírico de investigación, el cual, dada sus características, facilita el proceso de análisis de la labor docente desde el contexto en que esta se realiza, ya que el observador, desde un ángulo no participante, puede visualizar lo que sucede para identificar las fortalezas $y$ aspectos por mejorar que el observado manifiesta, y así apoyar su labor a partir de un diálogo posterior al proceso (p.65).

\subsection{Entrevistas}

Es una plática entre un entrevistador y uno o más entrevistados. Este recurso se lleva a cabo a partir de un cuestionario que puede ser: a) estructurado, donde el investigador efectúa cierta cantidad de preguntas a las que el entrevistador se sujeta de forma exclusiva; b) semiestructurado, donde se elaboran una serie de preguntas, pero con la diferencia de que el entrevistador puede formular más de acuerdo a temas que considera debe profundizar y c) no estructurado o abierto, donde se cuenta con una guía con las preguntas que se desean 
hacer pero el entrevistador tiene la libertad de modificar la estructura, contenido del mismo durante la entrevista (Hernández et al., 2010).

La entrevista es una técnica muy útil en la investigación cualitativa, ya que permite obtener datos. Se define como una interacción con un objetivo distinto al hecho de sólo platicar (Diccionario de Ciencias de la Educación, 1983).

Es un recurso técnico que toma la forma de una conversación coloquial. Canales la conceptualiza como la comunicación interpersonal establecida entre el investigador y el sujeto de estudio, a fin de obtener respuestas verbales a las interrogantes planteadas sobre el problema propuesto (Canales Cerón M, 2006).

Para Denzin y Lincoln (2005): la entrevista es "una conversación, es el arte de realizar preguntas y escuchar respuestas" (p. 643). Además, esta técnica está fuertemente influenciada por las características personales del entrevistador, así mismo, ha llegado a convertirse en una actividad de nuestra cultura, aunque la entrevista es un texto negociado, donde el poder, el género, la raza, y los intereses de clases han sido de especial interés en los últimos tiempos.

Se puede decir que la entrevista dentro de la investigación cualitativa es más íntima y manejable, según (Hernández et al., 2006), la entrevista de tipo cualitativa debe ser abierta, sin rangos establecidos previamente, de tal forma que los entrevistadores puedan hablar sobre sus experiencias.

Así mismo, Alonso (2007), nos señala que: "la entrevista de investigación es una plática entre dos individuos, un entrevistador y entrevistado, dirigida y registrada por el moderador, con el fin de beneficiar la producción de un discurso conversacional, con buenos argumentos y pocas segmentaciones" (p. 228). Por tanto, Según Fernández (s. f):

Es un modelo que propicia la integración dialéctica sujeto-objeto considerando las diversas interacciones entre la persona que investiga y lo investigado. Se busca comprender, mediante el análisis exhaustivo y profundo, el objeto de investigación dentro de un contexto único sin pretender generalizar los resultados. (p.91)

\subsection{Encuestas}

Según Hernández Sampieri (1997) la encuesta o cuestionario es el instrumento más utilizado para la recolección de datos, este se define como un documento formado por un conjunto de preguntas que deben estar redactadas de forma coherente, organizada, secuenciada y estructurada de acuerdo con una determinada planificación, con el fin de que sus respuestas nos puedan ofrecer toda la información necesaria (p.52).

\section{Conclusiones y recomendaciones}

A continuación, se detallan las conclusiones que se obtienen del trabajo de investigación: En relación con el objetivo específico número uno, elaborar un diagnóstico de las problemáticas ambientales presentes en cuatro instituciones educativas del distrito de Buenaventura. En el diagnostico se determinó que el $100 \%$ de las instituciones en las cuales se desarrolló la investigación presentan serios problemas en la gestión de los residuos, igualmente muestran deterioro en sus instalaciones físicas, y un sistema deficiente en acueducto, aseo y alcantarillado, existe fragmentación en el liderazgo de la gestión ambiental y poca participación de la dirección de las instituciones en los procesos, el cual recae específicamente en el área de ciencias naturales.

De otra parte, para este primer objetivo se encontró la poca integración o vinculación de los padres de familia a los procesos de gestión y educación ambiental en las cuatro instituciones educativas.

En cuanto al segundo objetivo específico, que buscaba establecer el alcance de los instrumentos del sistema de gestión ambiental basado en ISO 14001, para implementar en cuatro instituciones educativas del distrito de Buenaventura, se encontró que la implementación de la ISO 14001 en las instituciones presenta la dificultad de la estructura organizativa de los centros escolares en Colombia, que muchas veces son diferentes a la organización empresarial, las escuelas se distancian en su administración y propuesta del concepto empresarial, a pesar de que en muchos sectores se habla de empresas educativas, a nivel publico la estructura organizativa tiene unas particularidades como son: dependencia centralizada de las 
secretarias de educación, lo cual limita las decisiones, los procesos y el manejo de los rubros destinados a fortalecer la gestión ambiental en las instituciones educativas (En las cuatro instituciones no existen rubros asignados a los programas de educación ambiental), porque los colegios tienen una responsabilidad en la formación integral de sus estudiantes, aspecto por lo cual los procesos deben ir más allá de la mera gestión e involucrar acciones formativas; y tienen un factor adicional muy importante que son los actores padres de familia que juegan un papel fundamental en los procesos complementarios de formación, y se depende de su participación (activa o apática) la capacidad de convocatoria institucional y el respaldo que le den al colegio. Igualmente existe un desconocimiento de parte de los docentes en su gran mayoría (a excepción de algunos de ciencias naturales) de las normas ambientales, de las políticas de educación, de las estrategias de formación para la educación ambiental. Inclusive, en su gran mayoría han leído muy pocos documentos pedagógicos sobre el tema, finalmente se desconoce complemente sistema ISO 14001. Algunos creen que es de estricto cumplimiento.

Frente a lo anterior el alcance de la ISO 14001 para las cuatro instituciones educativas está sentado sobre las bases de una acción pedagógica centrada en Ecoauditorias ambientales, donde los estudiantes reconocen cuáles son los problemas ambientales institucionales y que lo importante no es el resultado, sino el proceso, además de una acción de gestión ambiental que gire en torno a la construcción de unos indicadores de evaluación de la gestión ambiental de la institución basada en la ISO 14001.

Para el tercer objetivo específico, que rezaba en proponer indicadores para un modelo de gestión ambiental, a partir de la ISO 14001 en cuatro instituciones educativas del distrito de Buenaventura, se diseñó un sistema de indicadores teniendo en cuenta tres variables que surgieron desde las categorías como son: gestión ambiental sostenible del entorno escolar, organización del centro y estructura del currículo, y participación de la comunidad educativa. A cada variable se le asignaron indicadores derivados de la ISO 14001 que pueden ser implementados en las instituciones educativas, se generaron unos ítems valorativos describiendo su significado y se definió su aplicación anual para verificar el avance en la gestión por parte de las instituciones.

\section{Referencias}

Alonso, L. E. (2007). Sujetos y Discurso: el lugar de la entrevista abierta en las prácticas de la sociología cualitativa. En J. M. Delgado y J. M Gutiérrez (Coord.). Métodos y técnicas cualitativas de investigación en Ciencias Sociales (pp. 225-240). Síntesis.

Álvarez, D. P. (2018). Propuesta de integración de los PRAES con la ISO 14001.

Angel, E., Carmona, S. I., \& Villegas, L. C. (2010). Gestión Ambiental en Proyectos de Desarrollo. Todograficas LTDA.

Arias, F. (2012). El proyecto de investigación: Introducción a la metodología científica. $\left(6^{a}\right.$ ed). Episteme.

Artaraz, M. (2001). Teoria de las tres dimensiones del Desarrollo Sostenible. Ecosistemas

Asenjo, R. (2006). Institucionalidad pública y gestión ambiental en Chile. Serie En Foco, 91.

Avellaneda, A. (2003). Gestión Ambiental y Planificación del Desarrollo. Reseñas

Ávila, B. M. (2016). La educación ambiental como alternativa para promover la convivencia ecológica. Revista scientific, 42- 53.

Canales Cerón, M. (2006). Metodologías de la investigación social. LOM Ediciones. (p.p. 163 165.).

Denzin, N. K., y Lincoln, Y. S. (2005). The Sage Handbook of Qualitative Research. London, Inglaterra: Sage.

Diccionario de Ciencias de la Educación (1983), Vol. 1. Santillana. (p. 208).

Hernández-Sampieri, R. (2014). Metodología de la Investigación $\sigma^{a}$ edición. Mc Graw Hill 
Sierra, B. (1984). Ciencias Sociales, Epistemología, Lógica y Metodología". Teoría y ejercicios. Paraninfo.

Badillo, M. (2012). Política de educación ambiental en Colombia.

Barrera, A. G. (2017). Las necesidades educativas especiales. Revista Ensaico.

Blanco, N., \& Pirela, J. (2016). Diseño de la investigación . Revista espacios públicos, pp. 97111.

Bedoya, Mocoso, A. V. (2015). Incidencia de los procesos educomunicativos en los proyectos ambientales escolares. Revista lasallista de investigación.

Bibiloni, H. M. (2008). Ambiente y política: Una visión integradora para gestiones viables. Editorial Rap.

Bifani, P. (1997). El desafío ambiental como un reto a los valores de la sociedad. Madrid: Fundación Universidad Empresa.

Bolzan, C., \& Pol, E. (2009). Sistemas de Gestión Ambiental y comportamiento ecológico: una discusión teórica de sus relaciones posibles. Aletheia .

Bordearías M. y Muguruza C. (2014) Evaluación ambiental. Editorial digital Uned. Madrid, España. Recuperado de: http://200.21.104.25/lunazul/index.php?option= com_content $\&$ view $=$ article $\& i d=219$

Bórquez, P, B, Lopicich, B. (2017). La dimensión bioética de los Objetivos de Desarrollo Sostenible (ODS)

Burgos, A, A. (2017). Estado de los proyectos ambientales escolares en Boyacá. Luna Azul, 44, 39-58. $\quad$ DOI: $\quad$ 10.17151/luaz.2017.44.4. Recuperado de: http://200.21.104.25/lunazul/index.php?option= com_content\&view $=$ article \&id $=219$

Caïs, J., Folguera, L., \&Formoso, C. (2014). Investigación cualitativa longitudinal (Vol. 52). CIS-Centro de Investigaciones Sociológicas.
Recuperado de https://books.google.com.co/books?id=oI6lBAA $\underline{A Q B A J \& p g=P A 7 \& d q=\text { longitudinal } \& h l=e s \& s a}$ $=X \& v e d=2 a h U K E w i Q g K 2 L$ -

MPsAhUjzlkKHdDsB2UQ6AEwAnoECAIQAg\# $v=$ onepage $\& q=$ longitudinal $\& f=$ false

Cáceres, M. A. (2016). Educación mabiental desde la la perspectiva cultural para la formación de profesionales en ciencias naturales. Luna Azul, 354-385.

Cáceres, R. Á. (2007). Estadística aplicada a las ciencias de la salud. Ediciones Díaz de Santos. Recuperado de https://books.google.com.co/books? id =V2Zosg PYIOkC\&pg $=$ PA219\&dq=poblacion $+y+$ muestr $a \& h l=e s \& s a=X \& v e d=2 a h U K E w j H 1 Z y J g 8 T_{s A}$ hVHmlkKHTR7CpUQ6AEwCXoECAkQAg\#v=O nepage \& $q=$ poblacion $\% 20 y \% 20$ muestra $\& f=$ fals $\underline{e}$

Catalina, M. M., \& Arturo, G. G. (2014). Técnicas e instrumentos de recogida y análisis de datos. Editorial UNED. Recuperado de https://books.google.com.co/books?id=iiTHAw $\underline{A A Q B A J \& \text { printsec }=\text { frontcover } \& d q=\text { instrument }}$ os + de + recolecci\%C3\%B3n+de+datos \& hl=es $\& s a=X \& v e d=2 a h U K E$ wiahKfwhcTsAhUSIlkK HQvkBJ8Q6AEwAXoECAYQAg\#v=onepage \&q $\underline{\& f=\text { false }}$

Caparó, E. V., García, V. R., \& Duffaut, A. C. (2016). Validación de cuestionarios. Revista Oactiva uc cuenca.

Calderón, J. P. (2010). La política ambiental en México: Gestión e instrumentos económicos. El Cotidiano.

Calvo, S. \& Corraliza, J. (1994). Educación Ambiental. Madrid: CCS. Conferencia Intergubernamental sobre Educación Ambiental, Tbilisi (1977). Recuperado de: http://unesdoc.unesco.org/images/0003/000327/ $\underline{032763 s b . p d f}$

Cervetto, A. M., \& Moreira, N. M. (2017). Diagnóstico del manejo de residuos sólidos. la granja: Revista de ciencias de la vida. 
Clements, R., 1997. Guía completa de las Normas ISO 14000. España. Ediciones Gestión 2000 S.A. p. 285

Collazos, D. C. (2019). Propuesta de formulacion de un sistema de gestión ambiental basado en la NTC ISO 14001 en el instituto Imety en el municipio de Yumbo.

Cárdenas, M., \& Rubiano, D. A. (2015). NTC ISO 14001 para el colegio Tomás Carrasquilla.

Cardona, O. D. (1996). Manejo Ambiental y Prevención de Desastres: Dos Temas Asociados . En M. A. Fernandez, Ciudades en Riesgos (pág. 57). Quito: La Red.

Castro, R. d. (2002). ¿Estamos dispuestos a proteger nuestro ambiente? Intención de Conducta y Comportamiento Proambiental. Medio Ambiente y Comportamiento Humano.

CEPAL. (1992). La variable población en la gestión ambiental: un ejemplo de evaluación de impacto ambiental. Santiago de Chile.

Cohen, M. (2007). Una nueva gestión ambiental: El riesgoy el principio precautorio. Cuaderno Venezolano de Sociología.

Corral Y. (2014). Instrumentos de recolección de datos: Validez y confiabilidad. EDITORIAL ACADEMICA ESPANOLA, España. PP. 1-88, 11.

Cuevas- Zuñiga, I., Rocha- Laana, L., \& SotoFlores, R. (2016). Incentivos motivacionales y beneficios de la incorporación de la gestión ambiental en las empresas. Universidad \& Empresa, 1-22.

Christian, T. (2015). Estrategias para usar racionalmente el agua. Industrial Data, 46-54.

DEL RÍO, S. D. (2013). Diccionario-glosario de metodología de la investigación social. Editorial UNED. Recuperado de https://books.google.com.co/books?id=XtlEAgA AQBAJ\&pg $=P T 258 \& d q=$ probabilistico \& $h l=e s$ $\& s a=X \& v e d=2 a h U K E w i 7 p Z 6 K \_c P s A h U M m 1 k$ KHTD5Cjg4ChDoATAFegQIBhAC\#v=onepage $\& q=$ probabilistico $\& f=$ false
Diaz, O., \& Meneses Silva, M. M. (2015). Criterios $e$ indicadores de sostenibilidad. Ciencias $e$ ingeniería Neogranadina.

Esteban, I. G., \& Fernández, E. A. (2017). Fundamentos y técnicas de investigación comercial. Esic Editorial. Recuperado de https://books.google.es/books?hl=es\&lr $=\& i d=z$ baaDgAAQBAJ\&oi=find\&pg $=P A 19 \& d q=F U N$ DAMENTOS $+Y+T E C N I C A S+D E+I N V e s t i g a c i$ on + comercial $+\&$ ots $=U 2 P M O I z H w 9 \&$ sig $=d 2-$ qiiDLMN4ynJVnlgG0aDnnPIk\#v=onepage \&q $=$ FUNDAMENTOS\%20Y\%20TECNICAS\%20D E\%20INVestigacion\%20comercial $\& f=$ false

Ferro Veiga J. M. (2020) Introducción al perito medioambiental para no iniciados. Pp 1-796, 17

Fornet Hernandez, E. (2020). Gestion ambiental organizacional en el centro de investigación y servicios ambientales Holguín. Ciencias Holguín.

Gallardo Martinez, D. (2019). Modelo de gestion ambiental integral. Minería y Geología, 1-18.

García J.P., (2005), Positivismo y Darwinismo. Ediciones Akal, S.A. Madrid pp. 17, 1-95

Gómez, P. A. (2016). Análisis de datos cualitativos. Revista Fedumar pedagogía y educación.

Gómez, M. M. (2006). Introducción a la metodología de la investigación científica. Editorial Brujas. Recuperado de https://books.google.es/books? hl=es\&lr=\&id=9 UDXPe4U7aMC\&oi=fnd\&pg $=P A 9 \& d q=$ intintr oducc $+a+$ la + metodologia + de + la + invesigacio $n+$ cientifica\&ots $=b 9 j H G T r S D V \&$ sig $=y 5 U U g-$ $n N 8 m M a A E s B I Q a \_l K 8 w O p s \# v=$ onepage $\& q=i n$ troduccion\%20a\%20la\%20metodologia\%22de $\% 20$ la\%20invesigacion\%20cientifica $\& f=$ false

Gonzales, E. (2001). Gestion Ambiental en Pequeños Municipios. Foro, 57.

Guerrero, A. B., \& Gutierrez, A. R. (2017). Evaluación del potencial emprendedor en escolar. Una investigación longitudinal. Revista Educación XX1, pp. 73-94. 
Henao Hueso, O., \& Sanchez Arce, L. (2019). Educación ambiental en colombia, utopía o realidad. Conrado, 213-219.

Hernández, A. (2016). Ciudad de México, al borde del colapso ambiental. Obtenido de Forbes: https://www.forbes.com.mx/243716-2/

Hurtado León, I. Toro garrido, J. (2007). Paradigmas $Y$ Métodos de Investigación en Tiempos de Cambios. Recuperado de https://books.google.com.co/books?id=pTHLXX Ma90sC\&pg=PA101\&dq=no+experimental \&hl $=e s \& s a=X \& v e d=2 a h U K E w j O l a 668 M P s A h V E j l$ kKHVhtDkwQ6AEwAnoECAQQAg\#v=onepage $\& q=n o \% 20$ experimental $\& f=$ false

Jaramillo, Carrillo, S, Alzate, $M, L \&$ Cardona $R, J$. D. (2017). Estudio exploratorio sobre las concepciones acerca de la educación ambiental de integrantes de la comunidad de dos establecimientos educativos del departamento de Antioquia.

Jiménez Madrid A. (2019) Auditorías Ambientales. Editorial elearning S.L. España. Pp 1-149, 8

José, N. A. M., Ángel, F. A., Concepción, G. E., Carlos, S. F. J., Ángeles, B. D., Guillermo, G. E., \& Encarnación, S. S. (2012). Métodos, diseños y técnicas de investigación psicológica. Editorial UNED. Recuperado de https://books.google.com.co/books?id=zbKzhys

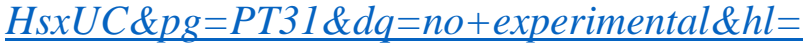

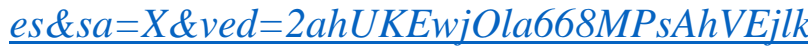
KHVhtDkwQ6AEwBXoECAMQAg\#v=onepage $\& q=n o \% 20$ experimental $\& f=$ false

La hoz Rodríguez E. (2020). Bases conceptuales, legislación y metodología de la evaluación de impacto ambiental. Editorial Elearning S.L. España. Pp. 1-323, 10

Lucie, S. (2004). Perspectivas curriculares para la formación de formadores en educacion ambiental.

Maestre A, Juan. (1989). La investigación en Antropología Social. Bogotá: Akal editor
Massolo, L. (2015). Introducción a las Herramientas de Gestión Ambiental. Mar de Plata. Pp.1-196, 11

Marin, M. I. (2011). Modelo de sistema de gestión ambiental. Gestión y ambiente, 151-162.

Martinez, J., \& Figueroa, A. (2013). Evolución de los conceptos y paradigmas que orientan la gestión ambiental ¿cuáles son sus limitaciones. Ingenierías, 13-27.

Mora, $O, R$ (2015). Los Proyectos Ambientales Escolares. Herramientas de gestión ambiental

Muñoz, Montilla, A. N. (2018). Propuesta de estructuración de un sistema de indicadores de educación ambiental. Revista colombiana de educación.

Muñoz-Montilla, A., \& Páramo-Bernal, P. (2018). Estructuración de un sistema de indicadores de educación ambiental. Revista colombiana de educación, 81-106.

Nannett, e. g., \& Leyva, P. (2015). La gestión ambiental en Colombia, 1994-2014: ¿un esfuerzo insostenible? Bogotá: Clima Gráfica.

Ochoa Ávila, M. B. (2016). Tecnología para la gestión ambiental integral en las instituciones escolares. Ciencias Holguín, 1-16.

Organización de las Naciones Unidas para la Alimentación y la Agricultura. (2015). La FAO y los 17 Objetivos de Desarrollo Sostenible

Ortega Y. y Garcia J. (2010). Un viaje por la educación ambiental en la escuela. Editorial edita. España. Pp 1-329, 22

Ortiz, J. R. (2015). Herramientas de gestión ambiental. Bitácora urbana Territorial, 67-74.

Oyaga, M, R. (2011). Construcción de indicadores de evaluación y estrategias de lúdica ambiental para la aplicación didáctica de las ecoauditorías ambientales escolares en instituciones educativas del distrito de barranquilla. Bogotá, Colombia. Coordinadora Editorial Revista educación e ingeniería. 
Pérez Juste R., Galán Gonzales A. \&Quintanal Díaz. (2012). Métodos y diseños de Investigación en Educación. J. Editorial UNED, Madrid Pp. 187 1-603

Perez Ramirez, L. (2017). Diseño de estructura de planeación para un sistema de gestión ambiental para el colegio nacional diversificado. Ingenio y sociedad.

Riojas, J., 2000. La complejidad ambiental en la Universidad. En Leff, E. (coord.), La complejidad ambiental, Siglo XXI- PNUMA, México. pp. 139- 215.

Rivera, J. A. (2020). Positivismo Pedagógico. Espiral.

Rocha, C. M. (2015). Metodología de la investigación. Oxford UniversityPress. Recuperado de https://books.google.com.co/books?id=DflcDw $A A Q B A J \&$ printsec $=$ frontcover $\& d q=$ hipotesis + de + investigacion $\& h l=e s \& s a=X \& v e d=2 a h U K E$ wjJg9K3i8TsAhVNulkKHfE4A_EQ6AEwBXoE CAkQAg\#v=onepage $\& q \& f=$ false

Rodilla Gonzales M. A. \& Ramón Pascua J.A., (2006) El positivismo jurídico a examen. Ediciones Universidad Salamanca, España pp. 767, 1-1296

Rodriguez, G. A. (2009). La participación en la gestión ambiental: Un reto para el nuevo milenio. Universidad del Rosario.

Rodriguez, M. (2011). Manuel Rodriguez Becerra. Obtenido http://www.manuelrodriguezbecerra.org/bajarl gestion/capitulo4.pdf

Rodríguez, R. G. (2016). Fundamentos básicos para la ejecución de la auditoría ambiental. Ciencias Holguín, 1-18.

Rojas Álvarez C.J. (2018). Razonamiento Cuantitativo Editorial Universidad del Norte, Barranquilla (pp 1, 1-113).

Ruiz, M. R., \& Pérez, E. S. (2014). Educacion ambiental en niños y niñas de instituciones educativas oficiales del distrito de santa marta. Zona próxima, 52-64.

Sacco, N., \& Andreozzi, L. (2017). Proyecciones y retroproyecciones probabilíticas de las tasas de fecundación por edad. Revista latinoamericana de población, pp. 79- 104.

Sallan Leys J. M. \& Fonollosa J. B. (2009) Métodos Cuantitativos de organización Industrial $I$. Ediciones UPC Barcelona. Pp 11, 1-214

Sampieri, R. H. (2018). Metodología de la investigación: las rutas cuantitativas,

cualitativa y mixta. McGraw Hill México. Recuperado de https://books.google.com.co/books? id =5A2QD $w A A Q B A J \& p g=P A 224 \& d q=$ Instrumentos $+d e+$

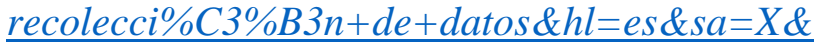
ved $=2$ ahUKEwjqjIacisTsAhVNmVkKHaOgAf84 ChDoATADegQIBhAC\#v=onepage \& $q=$ Instrum entos \%20de\%20recolecci\%C3\%B3n\%20de\%20 datos $\& f=$ false

Sauvé, L. (2003). Perspectivas curriculares para la formación de formadores en educación ambiental. I Foro Nacional sobre la Incorporación de la Perspectiva, (13). Luis de Potosí (México).

Suares-Silgado, S., Betancourt- Quiroga, C., Molina- Benavides, J., \& Mahecha-Schiffman L.G. \&Kanuk L.L, Comportamiento del consumidor. (2005) editorial Pestson, Pp 27, 1584

Steger, U., 2000. Environmental management systems: empirical evidence and further perspectives. EuropeanMangementJournal. $18(1): 23-37$

Teliz, E. G. (2020). Rutas de aprendizajes en la inducción, ingreso y seguimiento de un proceso de formación. Revista Educación.

Tiberius, J. (2016). Teoría cognitiva global. Molwick.

Tolley, E. E. (2006). Investigación aplicada en salud pública. Métodos cualitativos (No. 614). Pan American HealthOrg. 
Tomás-sábado, J. (2010). Fundamentos de bioestadística y análisis de datos para

enfermería (Vol. 2). Univ. Autónoma de Barcelona.

Recuperado

https://books.google.com.co/books?id=MHgap8

IN124C\&pg $=P A 21 \& d q=$ poblacion $+y+$ muestra

$\& h l=e s \& s a=X \& v e d=2 a h U K E w j H 1 Z y J g 8 T s A h$

VHmlkKHTR7CpUQ6AEwBXoECAEQAg\#v=o

nepage \& $q=$ poblacion $\% 20 y \% 20$ muestra $\& f=$ fals

$\underline{e}$

Torres Salas, T. C. (2015). Estrategia para uso racional del agua bajo la norma ISO 14001. Industrial Data, 46-54.

UNESCO, (1972). StockholmDeclaration.

Valdivieso, W. A. (2019). Ecoeficiencia: Nueva estrategia para la educación ambiental en las instituciones educativas. Sistema de información cientifica Redalyc, 77-84.

Vargas, A. T. (2015). Un modelo para la recolección de indicadores de calidad en los servicios gerontológicos. Revista Enseñanza e Investigación en psicología, pp. 56-68. 